\title{
Ellagic Acid Reduces High Glucose-Induced Vascular Oxidative Stress Through ERK1/2/ NOX4 Signaling Pathway
}

\author{
Artur Rozentsvit ${ }^{\mathrm{a}} \quad$ Kevin Vinokura $^{\mathrm{a}} \quad$ Sherin Samuel ${ }^{\mathrm{b}} \quad$ Ying Li $^{\mathrm{a}} \quad$ A. Martin Gerdes ${ }^{\mathrm{a}}$ \\ Maria Alicia Carrillo-Sepulveda ${ }^{a}$ \\ aDepartment of Biomedical Sciences, New York Institute of Technology, College of Osteopathic \\ Medicine, Old Westbury, NY, bDepartment of Life Sciences, New York Institute of Technology, Old \\ Westbury, NY, USA
}

\section{Key Words}

Ellagic acid $\cdot$ Antioxidant $\cdot$ High glucose $\bullet$ Endothelial dysfunction $\bullet$ Endothelial cell $\bullet$ NOX4 $•$ ERK1/2 • Diabetes

\begin{abstract}
Background/Aims: Elevated production of reactive oxygen species (ROS) is linked to endothelial dysfunction and is one of the key contributors to the pathogenesis of diabetic vascular complications. Emerging evidence has indicated that ellagic acid (EA), a polyphenol found in fruits and nuts, possesses numerous biological activities including radical scavenging. However, whether EA exerts a vasculo-protective effect via antioxidant mechanisms in blood vessels exposed to diabetic conditions remains unknown. Accordingly, the goal of this current study was to determine whether EA decreases vascular ROS production and thus ameliorates endothelial dysfunction in the diabetic milieu. Methods: Intact rat aortas and human aortic endothelial cells (HAEC) were stimulated with $30 \mathrm{mM}$ high glucose $(\mathrm{HG})$ with and without EA co-treatment. Endothelium-dependent vasodilation was measured using a wire myograph. Gene and protein expression of non-phagocytic nicotinamide adenine dinucleotide phosphate (NADPH) oxidases 4 (NOX4) were detected using RT-PCR and western blotting, respectively. Oxidative stress was determined by measuring ROS levels using dihydroethidium (DHE) staining. Results: Intact aortas exposed to HG condition displayed exacerbated ROS production and impairment of endothelium-dependent vasodilation, characterizing endothelial dysfunction. These effects were markedly reduced with EA treatment. HG enhanced ROS production in HAEC, paralleled by increased ERK1/2 activation and NOX4 expression. EA treatment blunted the increase of ROS generation, ERK1/2 activation and decreased NOX4. Conclusions: EA significantly decreases endothelial ROS levels and ameliorates the impairment of vascular relaxation induced by HG. Our results suggest that EA exerts a vasculo-protective effect under diabetic conditions via an antioxidant effect that involves inhibition of ERK1/2 and downregulation of NOX4.

A. Rozentsvit and K. Vinokur contributed equally to this work.




\section{Introduction}

Vascular complications are the leading cause of morbidity and mortality in both type 1 and type 2 diabetes, and remain one of the major health problems faced by the developed world. Hyperglycemia, characterized by high levels of blood glucose, is the primary contributor in the development of vascular dysfunction in diabetes. Studies from our group $[1,2]$ and others [3] have demonstrated that high glucose (HG) directly causes vascular dysfunction.

Under HG conditions, vascular endothelial cells (EC) generate high levels of ROS, inflammatory markers, and have impaired nitric oxide (NO) biosynthesis. Together these factors results in an imbalance between vasoconstriction and vasodilation, as well as an inability to regulate vascular tone characterizing endothelial dysfunction, the hallmark of diabetes [4-10]. HG leading to increased ROS-induced oxidative stress through PKCdependent activation of NAD(P)H oxidase in vascular EC [11] plays a central role in the onset of diabetic micro- and macrovascular disorders. Thus, targeting ROS generation has been considered a potential therapeutic target in diabetic patients.

In the last two decades, the use of anti-hyperglycemic drugs has markedly promoted optimal control of blood glucose, and substantially improved the quality of life for diabetic patients. However, these patients continue to present with macrovascular complications, including atherosclerosis, stroke, and coronary disease [12-14]. A possible explanation is that anti-hyperglycemic drugs may not decrease oxidative stress effectively. Antioxidant therapy has successfully decreased ROS levels, and improved endothelial dysfunction in an animal model and in type 1 diabetic patients $[8,15,16]$; however, large scale clinical trials have shown disappointing results with classic antioxidants, including vitamin $\mathrm{E}$, vitamin $\mathrm{C}$, and alpha-lipoic acid, failing to reduce the incidence of vascular complications in patients with type 2 diabetes $[17,18]$. These findings indicate that development of new alternative antioxidant approaches to target vascular ROS is needed.

Emerging epidemiological studies have shown an inverse relationship between cardiovascular disease mortality and diets rich in fruits and nuts in epidemiological studies [19-23]. High levels of many different phytochemicals, including polyphenols, are found in both fresh and processed fruits $[24,25]$. One such polyphenol is ellagic acid (EA) which is found in berries, grapes, pomegranates, and nuts. It has received increasing attention in the scientific community due its potential as an anti-inflammatory $[26,27]$ and antioxidant [28-30] compound. A recent study has demonstrated that EA lowered blood pressure in hypertensive rats via an antioxidant effect of EA which includes increased nitric oxide synthase (NOS) expression and decreased ROS levels in the plasma [28].

To date, whether EA exerts a protective role in vasculature exposed to high glucose conditions remains unknown. Accordingly, we hypothesize that EA reduces vascular ROS and consequently improves relaxation in blood vessels exposed to HG conditions through mechanisms that involves downregulation of NADPH oxidases. Results from this current study should provide important insights into the identification of EA as a new antioxidant strategy in preventing the development and/or retarding the progression of diabetes associated vascular complications.

\section{Materials and Methods}

Materials

Ellagic Acid (EA), glucose, acetylcholine (ACh), phenylephrine (PE), sodium nitroprusside (SNP), PD98059 and 2-hydroxyethidium (DHE) were purchased from Sigma (St. Louis, MO). Male Sprague Dawley rats were obtained from Charles River (New York, NY).

Animals

Male twelve-weeks-old Sprague-Dawley rats were used in ex vivo experimental procedures. Rats were maintained on a 12 hours light-dark cycle, fed standard rodent chow and drinking water ad libitium. 
All experiments and protocols were conducted in accordance with the National Institutes of Health (NIH) Guidelines for the Care and Use of Laboratory Animals and approved by the New York Institute of Technology College of Osteopathic Medicine (NYIT-COM) Animal Care and Use Committee.

\section{Organ culture of isolated aortas}

After euthanasia with isoflurane (via nasal 5\% in $100 \% \mathrm{O}_{2}$ ), thoracic aortas were removed and carefully cleaned of perivascular fat in oxygenated Krebs buffer $\left(130 \mathrm{mM} \mathrm{NaCl}, 14.9 \mathrm{mM} \mathrm{NaHCO}_{3}, 4.7 \mathrm{mM}\right.$ $\mathrm{KCl}, 1.18 \mathrm{mM} \mathrm{KH}_{2} \mathrm{PO}_{4}, 1.17 \mathrm{mM} \mathrm{MgSO}_{4}-7 \mathrm{H}_{2} \mathrm{O}, 1.56 \mathrm{mM} \mathrm{CaCl}_{2}-2 \mathrm{H}_{2} \mathrm{O}, 0.026 \mathrm{mM}$ EDTA, $5.5 \mathrm{mM}$ glucose, $\mathrm{pH}$ 7.4). Aortas were cut into rings ( $2 \mathrm{~mm}$ in length), cultured in Vascular Medium from ATCC (Manassas, VA), and maintained at $37^{\circ} \mathrm{C}$ in a $5 \% \mathrm{CO}_{2}$ incubator. Aortas were incubated with $30 \mathrm{mM}$ HG for 24 hours in the presence or absence of $20 \mathrm{uM}$ EA. Concentration of EA was chosen based on previous published reports [3133]. $5 \mathrm{mM}$ normal glucose (NG) was used as control. $25 \mathrm{mM}$ mannitol was utilized as an osmotic control for HG treatment. After treatment, some aortic rings were transferred to wire myograph for functional studies and some were embedded in optimal cutting tissue compound Tissue Tek OCT compound (Sakura Finetek, Terrance, CA) to measure ROS.

\section{Vascular Reactivity}

After treatment, aortic rings were mounted in a myograph for isometric tension recordings using a PowerLab 8/35 data acquisition system (ADInstruments Pty Ltd., Castle Hill, Australia), equilibrated with Krebs buffer for $30 \mathrm{~min}$, and gassed with $5 \% \mathrm{CO}_{2}$ in $95 \% \mathrm{O}_{2}$ at $37^{\circ} \mathrm{C}$, as described previously [34]. In all experiments, aortic ring integrity was assessed by stimulation with $120 \mathrm{mM} \mathrm{KCl}$. To test for the presence of endothelium, segments were contracted with $1 \mu \mathrm{M}$ PE and once the vessels reached a stable maximum tension, the vessels were stimulated with $10 \mu \mathrm{M}$ ACh and relaxation was confirmed (greater than $80 \%$ ). Cumulative concentration-response curves to $1 \mathrm{nM}-10 \mu \mathrm{M}$ ACh were performed in intact aortic rings previously incubated with 30mM HG for 24 hours in the presence or absence of EA. Endothelium-dependent relaxation was recorded for ACh after maximal pre-contraction with $1 \mu \mathrm{M} \mathrm{PE}$.

\section{Endothelial Cells Culture}

Human aortic endothelial cells (HAEC) were purchased from ATCC (Manassas, VA), grown in Endothelial Growth Medium (EGM-2MV) from Lonza (Portsmouth, $\mathrm{NH}$ ) and maintained at $37^{\circ} \mathrm{C}$ in a $5 \% \mathrm{CO}^{2}$ incubator. After reaching confluence, cells were starved for 24 hours in serum-free medium to reach a quiescent state. After this period, cells were pre-treated with $20 \mu \mathrm{M}$ EA (Sigma Aldrich, St. Louis, MO) and then stimulated with $30 \mathrm{mM}$ high glucose (HG) for 24 hours. Some set of experiments were performed in the presence of PD98059 (10 $\mu \mathrm{M}, 30$ minutes pre-incubation), a potent and selective ERK1/2 inhibitor [35]. As a control, HUVEC were maintained in $5 \mathrm{mM}$ normal glucose (NG). To rule out possible influence of osmotic stress, cells were also incubated in $25 \mathrm{mM}$ mannitol.

\section{Immunoblotting}

Total proteins were extracted from the cells and utilized for western blotting assay. After standard SDS-PAGE separation of proteins, samples were transferred to PVDF membranes, and immunoblotted with the following primary antibodies: NOX-4 (sc-30141, Santa Cruz Biotechnology, Santa Cruz, CA); NOX2 (ab129068, Abcam, Cambridge, MA); p47phox (sc-17845, Santa Cruz Biotechnology, Santa Cruz, CA); MnSOD (Millipore, Temecula, CA) and their respective secondary antibodies. Membranes were stripped and re-probed with an internal loading control, $\beta$-actin (\#4967, Cell Signaling, Danvers, MA). Protein bands were detected using ECL Plus (Amersham Biosciences, Piscataway, NJ). Data are presented as percent (\%) of control after normalized to $\beta$-actin.

\section{Quantitative real time PCR (RT-qPCR)}

Total RNA was extracted using Trizol from HAECs treated with HG and HG plus EA. Total RNA concentrations were determined and RNA was converted into cDNA using the SuperScript III First-Strand Synthesis System (Thermo Scientific). RT-qPCR (Bio-Rad iQ SYBR Green) was performed to analyze relative gene expression of NOX isoforms. According to the standard protocol for SYBR Green, the following primers were utilized: Human NOX1: AAGCCGACAGGCCACAGAT (forward), GTCACATACTCCACTGTCGTGTTTC (reverse). Human NOX2: GCAGCCTGCCTGAATTTCA (forward), TGAGCAGCACGCACTGGA (reverse). Human 
NOX4: CTTCCGTTGGTTTGCAGATT (forward), TGGGTCCACAACAGAAAACA (reverse). Human NOX5 AA- GACTCCATCACGGGGCTGCA (forward), CCCTTCAGCACCTTGGCCA- GAG (reverse). Human GAPDH: AGAAGGCTGGGGCTCATTTG (for- ward), AGGGGCCATCCACAGTCTTC (reverse). These primers, utilized for SYBR Green, were synthesized by Integrated DNA Technologies.

Measurement of reactive oxygen species (ROS)

Production of ROS was detected by $25 \mu \mathrm{M}$ DHE using method described previously [1]. Briefly, quiescent HAECs were pre-incubated with $20 \mu \mathrm{M}$ EA for 30 min followed by HG treatment for 12 hours. After that, $25 \mu \mathrm{M}$ DHE was added in the medium and incubated at $37^{\circ} \mathrm{C}$ for $20 \mathrm{~min}$. A subset of aortas treated with HG was simultaneously incubated with PEG-Catalase $(100 \mathrm{U} / \mathrm{mL})$ to identify the specificity of DHE to hydrogen peroxide $\left(\mathrm{H}_{2} \mathrm{O}_{2}\right)$. After incubation, HAECs were washed with PBS and fluorescence of DHE was detected using an Olympus DP73 fluorescence microscope fitted with a camera. In situ production of ROS of aortic rings treated for 24 hours with $\mathrm{HG}$ in the presence of $20 \mu \mathrm{M}$ EA was also determined using $25 \mu \mathrm{M}$ DHE. Aortic rings were embedded in Tissue Tek OCT compound (Sakura Finetek, Terrance, CA), slowly snapfrozen in isopentane cooled to $-80^{\circ} \mathrm{C}$. Cryosections of $5 \mu \mathrm{m}$ thickness were placed on Superfrost Plus slides (Menzel-Gläzer, Germany). $50 \mu \mathrm{L}$ of DHE solution was topically applied to each tissue section and the slide coverslipped. The slides were incubated in a light-protected and humidified chamber $\left(37^{\circ} \mathrm{C}, 30 \mathrm{~min}\right)$ before visualizing. Semi-quantitative analyses were performed to detect changes in fluorescence in HAECs or aortic rings using Image Pro Plus software.

\section{Statistical Analysis}

All data are expressed as mean \pm SEM. Concentration of ACh producing half-maximum relaxation (EC50) was expressed as - log EC50 (pD2 values). One-way repeated measures ANOVA followed by Bonferroni post hoc tests as utilized to compare vascular reactivity. Student's t-test was used when appropriated. $\mathrm{p}<0.05$ was considered statistically significant. The " $\mathrm{n}$ " in each experiment represents the different biological replicates.

\section{Results}

\section{Effect of EA on HG-induced impaired vasorelaxation}

Evidence shows that $\mathrm{HG}$ can directly impair vasorelaxation $[6,36]$. To determine whether long-term stimulation with HG impairs endothelium-dependent vasorelaxation, we performed cumulative concentration curves to ACh in intact aortic rings incubated with $30 \mathrm{mM} \mathrm{HG}$ for 24 hours. ACh-induced relaxation was significantly decreased in aortic rings incubated with HG (Fig. 1A), compared with those aortic rings vehicle control. HG significantly decreased sensitivity of the aortas to ACh, when compared to aortas control (pD2: $6.25 \pm 0.1$ vs $6.78 \pm 0.1$ control group). Together these vascular reactivity results confirm that HG directly causes endothelial dysfunction. To test our hypothesis that EA has a protective effect in the vasculature exposed to HG, vascular reactivity was performed in aortic rings incubated with HG for 24 hours in the presence or absence of $20 \mu \mathrm{M}$ EA. As shown in Fig. $1 \mathrm{~B}$, incubation of aortic rings with EA markedly reduced impaired vasorelaxation induced by $\mathrm{HG}$ and ameliorated sensitivity of the aortas, previously exposed to HG, to ACh (pD2: $6.70 \pm 0.1$ vs. $6.25 \pm 01 \mathrm{HG}$ group). EA treatment did not affect the ACh-induced vasorelaxation in aortic rings under NG, vehicle control group (Fig. 1C). These results suggest that EA plays a vascular protective role in blood vessels under hyperglycemic conditions.

\section{Ellagic Acid reduces HG-induced COX-2 expression}

EA has been suggested to possess anti-inflammatory effects that reduce vascular events [37]. Cyclooxygenase 2 (COX-2) is an inflammatory marker that is upregulated in diabetic vasculature. We next investigated the effect of EA on COX-2 expression in HAEC stimulated with HG. We found that exposure to HG increased COX-2 expression in HAEC. This effect was reversed by co-incubation with EA (Fig. 2). This result confirms that EA has an antiinflammatory effect in the vasculature exposed to hyperglycemic conditions. 
Fig. 1. Ellagic acid (EA) prevents HG-induced impaired endothelium vascular relaxation in rat aortas. Intact aortic rings were incubated with $30 \mathrm{mM} \mathrm{HG}$ for $24 \mathrm{~h}$ in the presence or absence of 20 $\mu \mathrm{M}$ EA through organ culture procedure. Concentration-response curves to ACh were performed in aortas incubated with HG (A), HG plus EA (B), control plus EA (C). Combined curves are represented in panel (D). Vehicle control group: aortic rings incubated in 5 mM NG. Data represent the mean \pm SEM of the values of relaxation (\% of PE-induced pre-contraction). $n=6$ per group. In the panel A, ${ }^{*} \mathrm{p}<0.05$ vs. vehicle-control. In the panel $B,{ }^{*} \mathrm{p}<0.05$ vs. HG group.

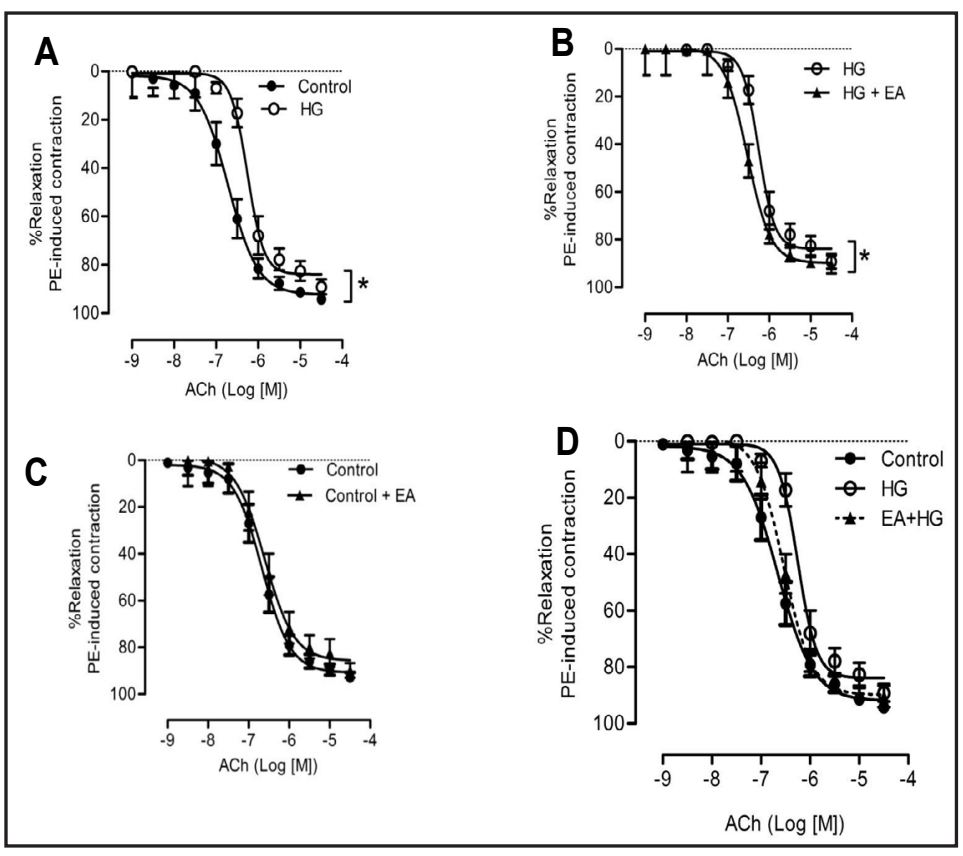

Ellagic Acid suppresses $H G$ induced vascular oxidative stress

To determine the effects of EA on oxidative stress, we measured ROS production in aortic rings and HAEC treated with HG in the presence and absence of EA. As shown in Fig. 3, exposure to $\mathrm{HG}$ for 24 hours led to ROS overproduction in rat aortas detected by DHE staining, which was significantly reduced in the presence of EA. Staining for DHE was abolished when simultaneously incubated with PEG-Catalase, indicating $\mathrm{H}_{2} \mathrm{O}_{2}$ production (Fig. 3). To confirm that EC are the main source of HG-induced ROS production, HAEC were stimulated with HG in the presence and absence of EA. Likewise observed in the aortic rings, HAEC also exhibited overproduction of ROS when stimulated with $\mathrm{HG}$, which was reduced in the presence of EA (Fig. 4). HG-induced increased DHE staining was abolished when simultaneously incubated with PEG-Catalase, confirming $\mathrm{H}_{2} \mathrm{O}_{2}$ production (Fig. 4).

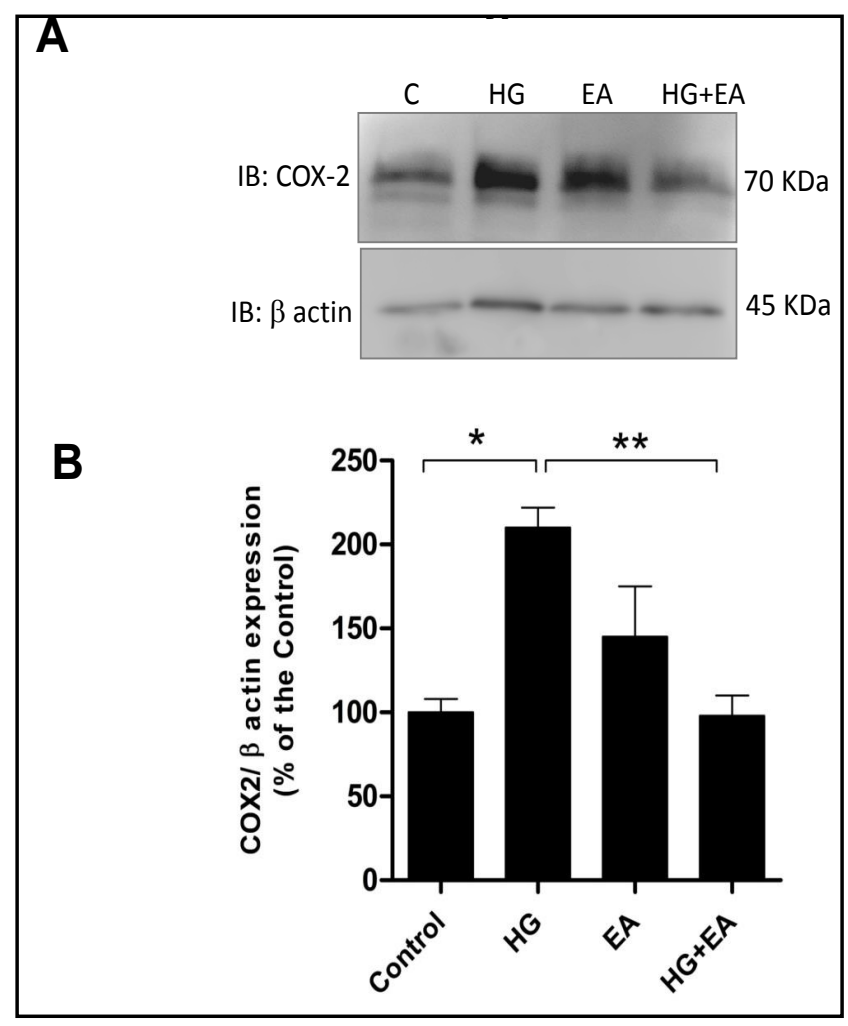

Fig. 2. EA reduces HG-induced COX-2 expression, a marker of inflammation, in HAEC. Representative immunoblot of COX-2 protein (A). Western blot analysis by densitometry (B). $\beta$ actin was used as a loading control. $n=4,{ }^{*} \mathrm{p}<0.05$ vs. control group; **vs. HG group. 
Rozentsvit et al.: High Glucose-Induced Vascular Dysfunction and Vascular Protective Effect of Ellagic Acid

Fig. 3. EA reduces elevated aortic ROS production induced by $\mathrm{HG}$ as indicated by changes in DHE fluorescence. (A) Representative photomicrographs depicting DHE fluorescence in aortic ring treated with $\mathrm{HG}$ and HG + EA. (B) Quantification of ROS was determined through fluorescent intensity in each experimental group. Results represent mean \pm SEM. $n=4$ per group, ${ }^{*} \mathrm{p}<0.05$ vs. control group; $* *$ $\mathrm{p}<0.05$ vs. HG group;

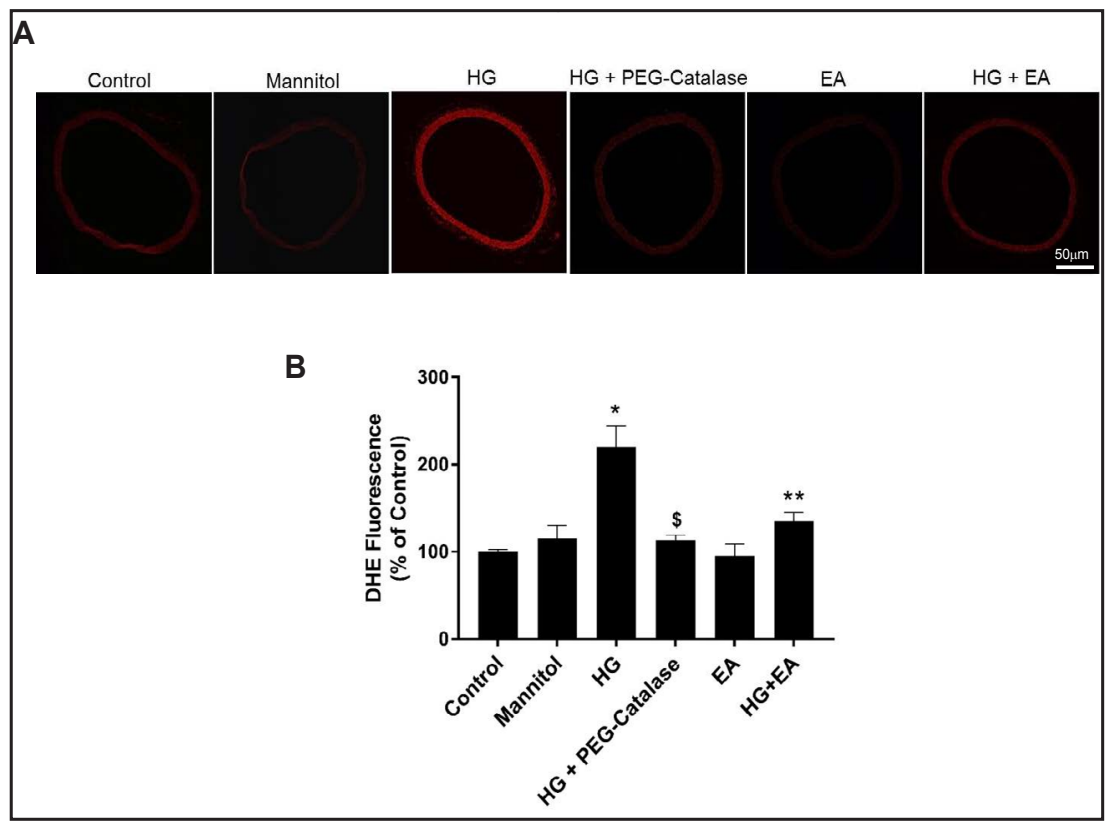
$\$ \mathrm{p}<0.01$ vs. HG group.

Fig. 4. EA reduces HGinduced ROS overproduction in HAEC. Quiescent HAEC were pretreated with $20 \mu \mathrm{M}$ EA, followed by stimulation with $30 \mathrm{mM} \mathrm{HG}$ for 30 minutes. ROS production was detected using DHE staining. Top panel: Representative photomicrographs depicting DHE fluorescence in each experimental group. Bottom panel: quantification of DHE staining was determined through fluorescent intensity in each cell

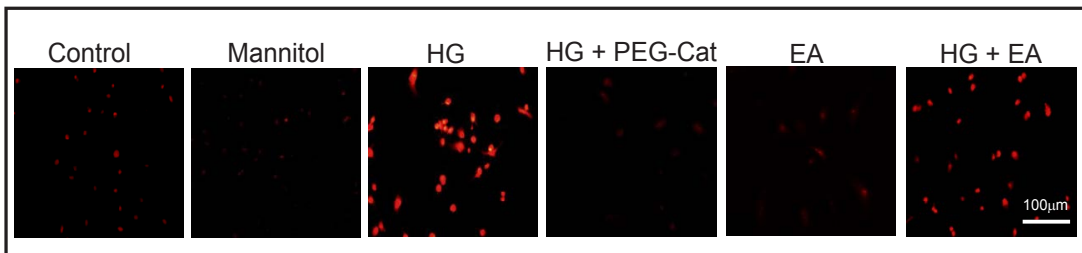
by pixel intensity of

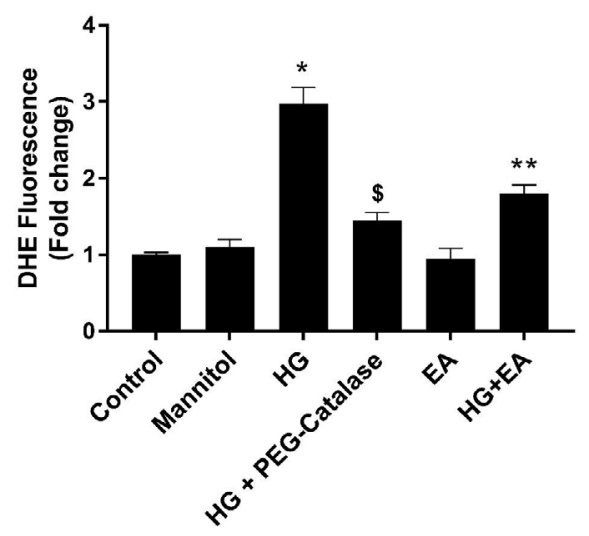

the cell. Results represent mean \pm SEM. $n=4$ per group, ${ }^{*} \mathrm{p}<0.05$ vs. control group; ${ }^{* *} \mathrm{p}<0.05$ vs. HG group; $\$$ $\mathrm{p}<0.05$ vs. HG group.

\section{Ellagic acid reduces expression of NOX4}

Vascular complications in diabetes are associated with HG-induced increased ROS levels. NADPH oxidases (NOX) are the major source of vascular ROS, including NOX1, NOX2, NOX4 and NOX5 [38]. Thus, we next analyzed the gene expression of NOX isoforms in HAEC stimulated with HG. As shown in Fig. 5, HG caused a 2-fold increase of NOX4 and 1.5-fold increase in NOX2 gene expression. Treatment with EA markedly decreased NOX4 gene but not NOX2 gene expression. All others NOXs were not altered by HG and EA treatment (Fig. 5). At protein levels, EA treatment also decreased significantly NOX4 expression (Fig. 6).

\section{KARGER}




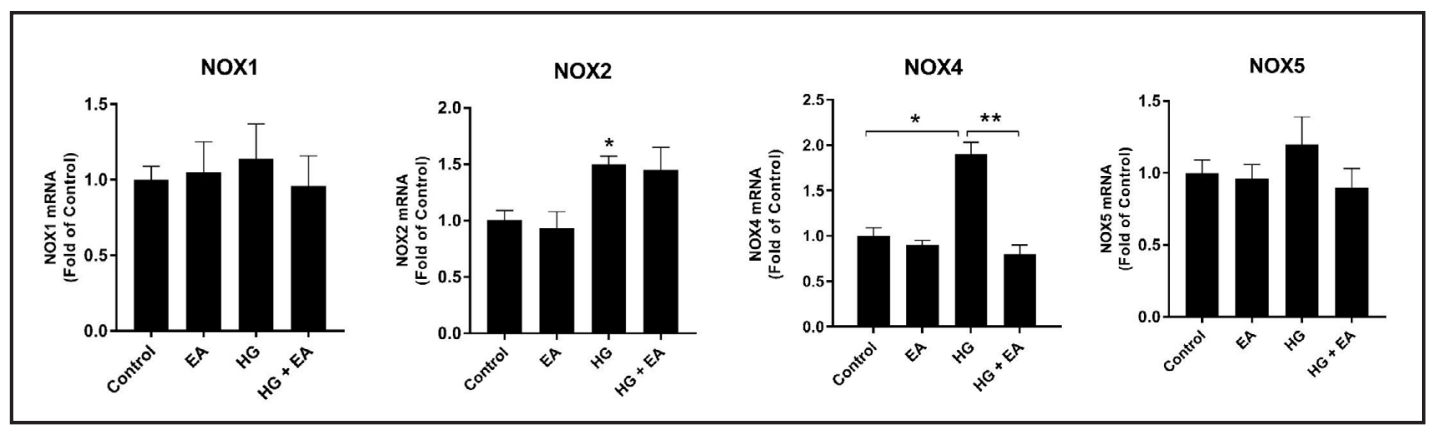

Fig. 5. Effect of EA in the mRNA of NOX1, NOX2, NOX4 and NOX5 in HAEC under HG conditions. HAEC were treated with 30mM HG for $24 \mathrm{~h}$ in the presence or absence of $20 \mu \mathrm{M}$ EA. After that, NOX1, NOX2, NOX4 and NOX5 mRNA levels were determined using RT-PCR relative to GAPDH (A). Results are representative of 4 separate experiments. ${ }^{*} \mathrm{p}<0.05$ vs. control group; ${ }^{* *} \mathrm{p}<0.05$ vs. HG group.

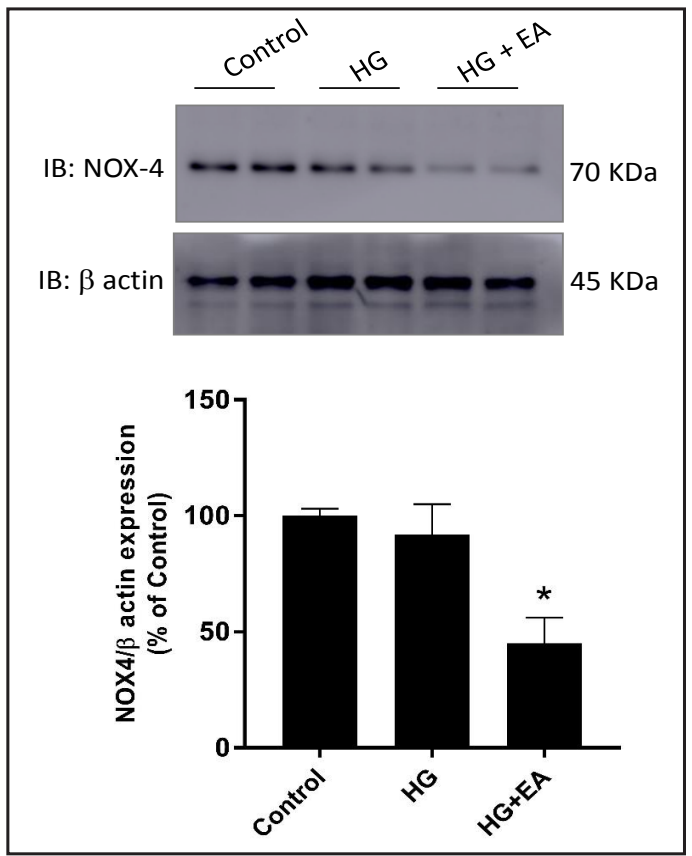

Fig. 6. Effect of EA in protein expression of NOX4 in HAEC under HG conditions. HAEC were treated with $30 \mathrm{mM} \mathrm{HG}$ for $24 \mathrm{~h}$ in the presence or absence of $20 \mu \mathrm{M}$ EA. After that, NOX4 protein expression was determined using western blot, with $\beta$ actin used as a loading control (B). Results are representative of 4 separate experiments. ${ }^{*} \mathrm{p}<0.05$ vs. HG group.

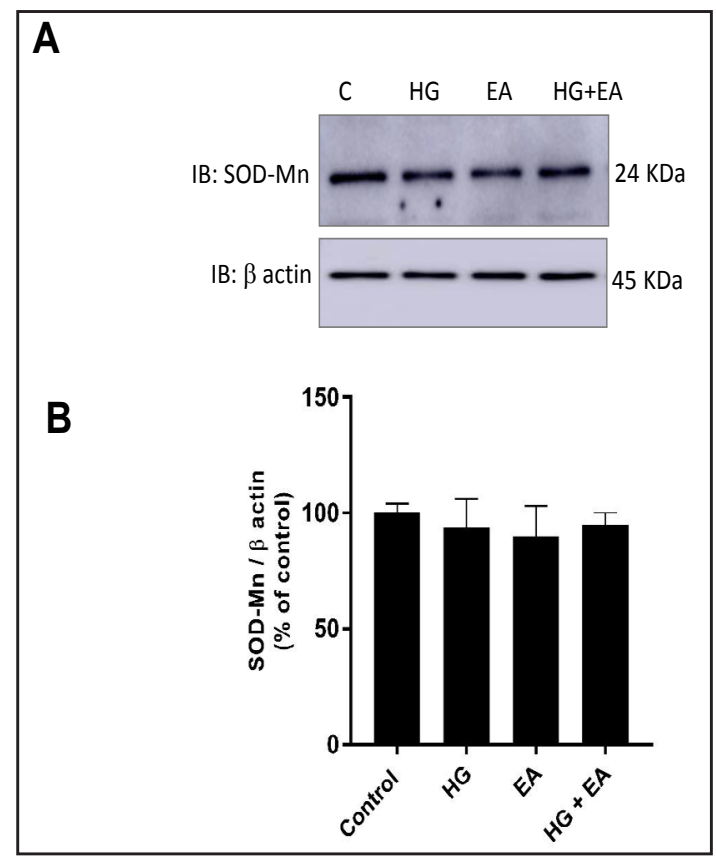

Fig. 7. Effect of EA in SOD-Mn expression, an antioxidant enzyme. HAEC were treated with 30 $\mathrm{mM} \mathrm{HG}$ for $24 \mathrm{~h}$ in the presence or absence of $20 \mu \mathrm{M}$. Representative immunoblot of SOD-Mn protein (A). Western blot analysis by densitometry (B). $\beta$-actin was used as a loading control. $n=4$.

We also checked the p47phox expression, a possible source of vascular ROS. No changes were observed with EA treatment (data not shown). EA treatment did not show any effect in antioxidant enzymes, such as MnSOD (Fig. 7).

\section{Ellagic acid inhibits $H G$-induced ERK1/2 activation}

HG activates MAPKs, mainly ERK1/2 [39, 40]. In order to determine whether EA inhibits HG-induced ERK1/2 activation and consequently NOX4, we examined the levels of

\section{KARGER}


Fig. 8. Effect of EA in ERK1/2 signaling pathway in HAEC under HG conditions. HAEC were treated with $30 \mathrm{mM} \mathrm{HG}$ for $24 \mathrm{~h}$ in the presence or absence of $20 \mu \mathrm{M}$ EA. Some cells were also treated with $10 \mu \mathrm{M}$ PD98059. After that, phosphorylated ERK1/2 levels were determined using western blot. $\beta$ actin was used as a loading control. Top panel: representative immunoblot of phosphorylated ERK1/2. Bottom panel: Western blot analysis by densitometry. $n=4$, ${ }^{*} \mathrm{p}<0.05$ vs, control group; ${ }^{* *}$ vs. HG group.

phosphorylated ERK1/2 in HAEC stimulated with $\mathrm{HG}$ in the presence and absence of EA. PD98059 $(10 \mu \mathrm{M}, 30$ minutes pre-incubation) [41], a potent and selective inhibitor of ERK1/2 [42], was used as a positive control. As shown in Fig. 8, HG increased phosphorylation of ERK1/2 in HAEC. This effect was significantly reduced by EA and PD98059, confirming that EA reduces $\mathrm{HG}$-induced ERK1/2 activation in endothelial cells. These results together with our data demonstrating that EA reduced NOX4 expression (Fig. 5 and Fig. 6) suggest

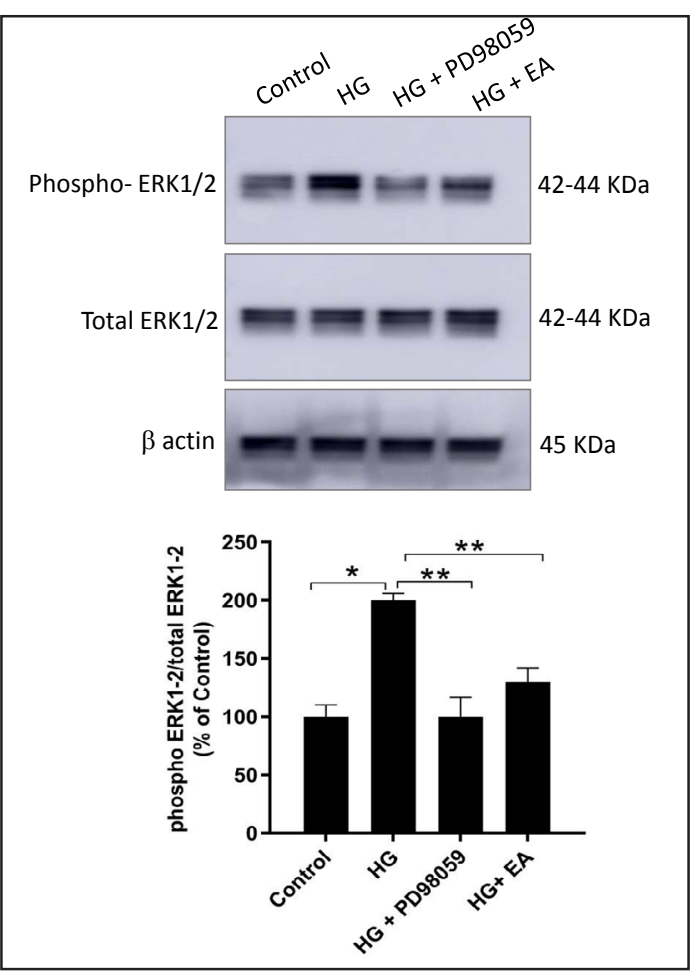
that EA exerts its vascular anti-oxidant effects via inhibition of ERK1/2/NOX4 axis pathway.

\section{Discussion}

Oxidative stress is a major contributor towards development and progression of diabetic vascular complications. Intriguingly, most of the conventional antioxidant agents tested in clinical trials have failed to avert the advancement of cardiovascular complications, indicating an urgent need to investigate other potential antioxidants as an alternative therapy. The main findings of this current study are that EA: 1) ameliorates the impaired relaxation induced by HG conditions and 2) markedly decreases endothelial redox machinery, as evidenced by reduced $\mathrm{H}_{2} \mathrm{O}_{2}$ production in vessels exposed to HG. This effect was accompanied by decreases in NOX4 expression, one of the major sources of vascular ROS. The present study provides evidence for EA as a potent vascular antioxidant agent and suggests that NOX4 may be a major target of EA. Unlike other antioxidant therapies, EA also possess an anti-inflammatory effect, which shows its potential as an anti-inflammatory and antioxidant therapy to treat vascular complications in diabetes. The novelty of our study lies in elucidating the mechanism by which EA exerts an antioxidant effect in a hyperglycemic vascular environment designed to simulate diabetic conditions.

Hyperglycemic conditions lead to increased oxidative stress and production of proinflammatory mediators on vascular cells resulting in dysfunction of vascular relaxation/ contraction mechanisms [43]. Importantly, some of these effects result from a direct effect of $\mathrm{HG}$ on the vasculature. For example, Wu et al. demonstrated that $55 \mathrm{mM}$ HG directly impaired vasorelaxation [36]. Consistent with these earlier reports, we also found that aortas incubated with $30 \mathrm{mM} \mathrm{HG}$, a concentration which mimics glucose levels more closely to that found in diabetic patients with poor glucose control, also displayed impaired endotheliumdependent vasorelaxation. Therefore, these results demonstrate that HG has the ability to directly cause endothelial dysfunction. 
Besides HG as a trigger of diabetic vascular dysfunction, the crosstalk between EC and vascular smooth muscle cells (VSMC) also plays a crucial role in promoting ROS overproduction and vascular dysfunction $[43,44]$. Previous studies have shown that EC exposed to HG display decreased nitric oxide (NO) and increased ROS production [11, 45, 46]. This imbalance between NO and ROS causes marked functional impairment of the vascular relaxation/contraction machinery. As expected, our results from human EC and intact rat aortas revealed that HG significantly increases vascular ROS production. Of note, HG also stimulates ROS production in VSMC as we demonstrated previously [47]. This augmented oxidative state in VSMC promoted by overproduction of ROS derived from EC and VSMC leads to VSMC phenotypic modulation, which is characterized by a switch from a native contractile phenotype to a synthetic proliferative phenotype, resulting in vascular remodeling [43]. Taken together, these results show that contribution of both EC and VSMC is essential for the increased vascular oxidative stress induced by hyperglycemia.

Interestingly, clinical observations have revealed that even in patients with optimal control of blood glucose, vascular complications continue to occur at a higher incidence when compared to the non-diabetic population [48]. One plausible explanation for this phenomenon is that glucose control does not necessarily avert vascular oxidative stress. This has led to the understanding that the production of ROS and increased oxidative stress plays a key role in the pathogenesis of diabetic vascular complications [44], clearly indicating that reduction of oxidative stress through antioxidant treatment is imperative. However, clinical trials such as the Heart Outcomes Prevention Evaluation (HOPE), the largest trial for the use of antioxidants in diabetes, have demonstrated that most of the conventional antioxidants do not confer clinical benefits to type 2 diabetic patients with cardiovascular complications $[16,49,50]$. Importantly, these trials were not specifically designed to investigate the effect of antioxidants in diabetic patients with vascular complications. Likewise, recent clinical trials testing some antioxidants, such as Resveratrol and Quercetin, which have previously demonstrated benefits in animal and in vitro experimental models, have not shown the same positive results in humans [51].

NOX are a major source of ROS in the vasculature, and includes NOX1, NOX2, NOX4 and NOX5 isoforms. In the present study, we found that NOX2 [52] and NOX4 were significantly upregulated by the HG state [53]. Further, we investigated which NOX isoforms may be modulated by EA treatment. Surprisingly, our results showed that NOX4 was the only isoform markedly downregulated by EA treatment, suggesting that NOX4 is the vascular antioxidant target of EA. Furthermore, ROS production detected by DHE staining was abolished in the presence of PEG-Catalase, indicating that $\mathrm{H}_{2} \mathrm{O}_{2}$ production in hyperglycemic conditions. Together, these results confirm that EA inhibits NOX $4 / \mathrm{H}_{2} \mathrm{O}_{2}$ axis.

Similarly, other studies have found that drugs with antioxidant properties exert their effects in the cardiovascular system via modulation of NOX4. For example, hydrogen sulfide $\left(\mathrm{H}_{2} \mathrm{~S}\right)$ exerts its antioxidant effects in cerebrovascular dysfunction via mechanisms that involves downregulation of NOX4 expression [54]. Statins, a lipid-lowering medication, has been reported to exert an antioxidant effect through inhibition of NOXs [55]. In the same manner, a recent study investigating alternative therapeutic approaches to treat nephrotoxicity found that thymoquinone significantly diminished oxidative stress-induced renal tissue damage and this was associated with decreased NOX4 activity [56]. Moreover, reports have shown that increased oxidative stress in cardiovascular disease in aging is mediated mainly by NOX4 [57]. Studies in diabetic mice with nephropathy have also shown that overgeneration of renal ROS is associated with upregulated NOX4 in the kidney cortex upon exposure to high glucose [58]. Increasingly, NOX4 has been proven to be one of the main sources of ROS in vascular disease, suggesting that targeting NOX4 activity and expression may be a potential approach to reduce vascular oxidative stress $[59,60]$.

Accumulating evidence suggests that high glucose activates MAPKs, mainly ERK1/2. Most remarkably, it was previously demonstrated that activation of ERK1/2 by diabetic stimuli, such as ox-LDL, directly induces NOX4 expression [61]. In this present study we hypothesized 
that EA downregulates HG-induced NOX4 overexpression via interference in ERK/1/2 signaling pathway. Our results demonstrated that EA significantly inhibited HG-induced ERK1/2 activation in endothelial cells. These findings are in accordance with other studies that have demonstrated that polyphenols, such as resveratrol, causes its beneficial effects via inhibition of the ERK1/2 molecular pathway [62].Therefore, we speculate that EA exerts its vascular anti-oxidant effects via inhibition of ERK1/2/NOX4/ROS axis pathway (Fig. 9).

Together with oxidative stress effects, inflammation also plays a crucial role in the vascular complications in diabetic populations [63]. Studies have demonstrated that there is an increase in

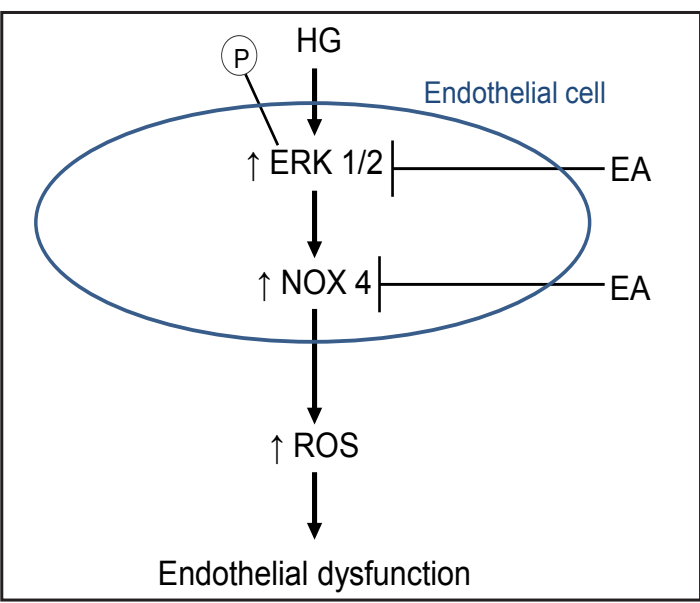

Fig. 9. Schematic summary of the present study. Ellagic acid (EA) exerts its anti-oxidant effect in endothelial cells exposed to HG via inhibition of ERK1/2/NOX4 signaling pathway. serum and tissue inflammatory markers and mediators in the hyperglycemic state [64], and that hypoglycemic agents exert an anti-inflammatory effect in diabetic patients [65]. EA itself has been studied for its anti-inflammatory effects and previous studies have shown that EA suppressed the production of cytokines, prostaglandins, and other inflammatory markers [66]. In our present study, we have shown that EA decreased expression of COX2, an inflammatory marker, in cells exposed to HG conditions [67].

While the anti-inflammatory effects of EA are well documented [67], few studies have examined the antioxidant effects of EA [29,30] and its possible role in novel therapeutics for cardiovascular complications. Here we report that EA exerts antioxidant and antiinflammatory effects in vasculature previously exposed to HG conditions. These findings provide novel insight into the antioxidant effects of EA in blood vessels under diabetic conditions. Exposure of vascular cells and blood vessels to glucose concentrations ranging from $4.2 \mathrm{~g} / \mathrm{L}(23.1 \mathrm{mM})$ to $10 \mathrm{~g} / \mathrm{L}(55 \mathrm{mM})$ for 24 hours resembles the diabetic milieu within cell culture systems [36, 68-71] and closely mimics a hyperglycemic environment in the vasculature of individuals with diabetes. Several studies have shown that endothelial dysfunction arises even after short-term hyperglycemic injury is inflicted. Shimabukuro et al. demonstrated that one single loading dose of a high glucose meal impaired endothelial function in type 2 diabetic patients [72].

Ultimately, our results support EA as an antioxidant that may provide benefits to treat diabetic vascular dysfunction. Dietary supplementation with EA has the potential to be utilized as a complementary therapeutic approach in conjunction with the use of current anti-hyperglycemic drugs to treat diabetic patients with high risk to develop vascular complications. These data support further investigation into the clinical possibilities of EA in diabetes care.

\section{Abbreviations}

EA (ellagic acid), HAEC (human aortic endothelial cells), HG (high glucose), NO (nitric oxide), NOX (non-phagocytic nicotinamide adenine dinucleotide phosphate (NADPH) oxidases), ROS (reactive oxygen species), VSMC (vascular smooth muscle cells). 


\section{Acknowledgements}

This work was supported by Faculty Development Grant from NYITCOM (MACS) and the National Heart, Lung, and Blood Institute of the National Institutes of Health, Bethesda MD, under award R01HL103671 (AMG). The content is solely the responsibility of the authors and does not necessarily represent the official views of the National Institute of Heath.

\section{Disclosure Statement}

None.

\section{References}

1 Carrillo-Sepulveda MA, Spitler K, Pandey D, Berkowitz DE, Matsumoto T: Inhibition of TLR4 attenuates vascular dysfunction and oxidative stress in diabetic rats. J Mol Med (Berl) 2015;93:1341-1354.

2 Schmidt L, Carrillo-Sepulveda MA: Toll-like receptor 2 mediates vascular contraction and activates RhoA signaling in vascular smooth muscle cells from STZ-induced type 1 diabetic rats. Pflugers Arch 2015;467:2361-2374.

3 Wu N, Shen H, Liu H, Wang Y, Bai Y, Han P: Acute blood glucose fluctuation enhances rat aorta endothelial cell apoptosis, oxidative stress and pro-inflammatory cytokine expression in vivo. Cardiovasc Diabetol 2016;15:109.

4 Pahwa R, Nallasamy P, Jialal I: Toll-like receptors 2 and 4 mediate hyperglycemia induced macrovascular aortic endothelial cell inflammation and perturbation of the endothelial glycocalyx. J Diabetes Complications 2016;30:563-572.

5 Chen S, Yang J, Xiang H, Chen W, Zhong H, Yang G, Fang T, Deng H, Yuan H, Chen AF, Lu H: Role of sphingosine-1-phosphate receptor 1 and sphingosine-1-phosphate receptor 2 in hyperglycemia-induced endothelial cell dysfunction. Int J Mol Med 2015;35:1103-1108.

6 Guo X, Liu WL, Chen LW, Guo ZG: High glucose impairs endothelium-dependent relaxation in rabbit aorta. Acta Pharmacol Sin 2000;21:169-173.

7 Wang LP, Jiang Y, Yang H, Peng C, Zhang C, Tao X, Xie HH: Combination Therapy of Nifedipine and Sulphonylureas Exhibits a Mutual Antagonistic Effect on the Endothelial Cell Dysfunction Induced by Hyperglycemia Linked to Vascular Disease. Cell Physiol Biochem 2016;38:2337-2347.

8 Sena CM, Pereira AM, Seica R: Endothelial dysfunction - a major mediator of diabetic vascular disease. Biochim Biophys Acta 2013;1832:2216-2231.

-9 Creager MA, Luscher TF, Cosentino F, Beckman JA: Diabetes and vascular disease: pathophysiology, clinical consequences, and medical therapy: Part I. Circulation 2003;108:1527-1532.

-10 Luscher TF, Creager MA, Beckman JA, Cosentino F: Diabetes and vascular disease: pathophysiology, clinical consequences, and medical therapy: Part II. Circulation 2003;108:1655-1661.

-11 Inoguchi T, Li P, Umeda F, Yu HY, Kakimoto M, Imamura M, Aoki T, Etoh T, Hashimoto T, Naruse M, Sano H, Utsumi H, Nawata H: High glucose level and free fatty acid stimulate reactive oxygen species production through protein kinase C--dependent activation of NAD(P)H oxidase in cultured vascular cells. Diabetes 2000;49:1939-1945.

12 Beckman JA, Creager MA, Libby P: Diabetes and atherosclerosis: epidemiology, pathophysiology, and management. JAMA 2002;287:2570-2581.

-13 Zannad F, Cannon CP, Cushman WC, Bakris GL, Menon V, Perez AT, Fleck PR, Mehta CR, Kupfer S, Wilson C, Lam H, White WB, Investigators E: Heart failure and mortality outcomes in patients with type 2 diabetes taking alogliptin versus placebo in EXAMINE: a multicentre, randomised, double-blind trial. Lancet 2015;385:2067-2076.

14 Green JB, Bethel MA, Armstrong PW, Buse JB, Engel SS, Garg J, Josse R, Kaufman KD, Koglin J, Korn S, Lachin JM, McGuire DK, Pencina MJ, Standl E, Stein PP, Suryawanshi S, Van de Werf F, Peterson ED, Holman RR, Group TS: Effect of Sitagliptin on Cardiovascular Outcomes in Type 2 Diabetes. N Engl J Med 2015;373:232242. 


\section{Cellular Physiology Cell Physiol Biochem 2017;44:1174-1187 \begin{tabular}{l|l|l} 
DOI: 10.1159/000485448 & $\begin{array}{l}\text { (c) } 2017 \text { The Author(s). Published by S. Karger AG, Basel } \\
\text { www.karger.com/cpb }\end{array}$
\end{tabular}

Rozentsvit et al.: High Glucose-Induced Vascular Dysfunction and Vascular Protective Effect of Ellagic Acid

15 Kim H, Yun J, Kwon SM: Therapeutic Strategies for Oxidative Stress-Related Cardiovascular Diseases: Removal of Excess Reactive Oxygen Species in Adult Stem Cells. Oxid Med Cell Longev 2016;2016:2483163.

16 Beckman JA, Goldfine AB, Gordon MB, Garrett LA, Keaney JF, Jr., Creager MA: Oral antioxidant therapy improves endothelial function in Type 1 but not Type 2 diabetes mellitus. Am J Physiol Heart Circ Physiol 2003;285:H2392-2398.

17 Ceriello A: New insights on oxidative stress and diabetic complications may lead to a "causal" antioxidant therapy. Diabetes Care 2003;26:1589-1596.

18 Yim S, Malhotra A, Veves A: Antioxidants and CVD in diabetes: where do we stand now. Curr Diab Rep 2007;7:8-13.

19 Ros E: Health benefits of nut consumption. Nutrients 2010;2:652-682.

20 Wang X, Ouyang Y, Liu J, Zhu M, Zhao G, Bao W, Hu FB: Fruit and vegetable consumption and mortality from all causes, cardiovascular disease, and cancer: systematic review and dose-response meta-analysis of prospective cohort studies. BMJ 2014;349:g4490.

21 Hu D, Huang J, Wang Y, Zhang D, Qu Y: Fruits and vegetables consumption and risk of stroke: a metaanalysis of prospective cohort studies. Stroke 2014;45:1613-1619.

22 Larsson SC, Virtamo J, Wolk A: Total and specific fruit and vegetable consumption and risk of stroke: a prospective study. Atherosclerosis 2013;227:147-152.

23 Stackelberg 0, Bjorck M, Larsson SC, Orsini N, Wolk A: Fruit and vegetable consumption with risk of abdominal aortic aneurysm. Circulation 2013;128:795-802.

24 Seeram NP, Adams LS, Henning SM, Niu Y, Zhang Y, Nair MG, Heber D: In vitro antiproliferative, apoptotic and antioxidant activities of punicalagin, ellagic acid and a total pomegranate tannin extract are enhanced in combination with other polyphenols as found in pomegranate juice. J Nutr Biochem 2005;16:360-367.

25 de Brito Alves JL, de Sousa VP, Cavalcanti Neto MP, Magnani M, Braga VA, da Costa-Silva JH, Leandro CG, Vidal H, Pirola L: New Insights on the Use of Dietary Polyphenols or Probiotics for the Management of Arterial Hypertension. Front Physiol 2016;7:448.

-26 Tang B, Chen GX, Liang MY, Yao JP, Wu ZK: Ellagic acid prevents monocrotaline-induced pulmonary artery hypertension via inhibiting NLRP3 inflammasome activation in rats. Int J Cardiol 2015;180:134-141.

-27 Ahad A, Ganai AA, Mujeeb M, Siddiqui WA: Ellagic acid, an NF-kappaB inhibitor, ameliorates renal function in experimental diabetic nephropathy. Chem Biol Interact 2014;219:64-75.

28 Berkban T, Boonprom P, Bunbupha S, Welbat JU, Kukongviriyapan U, Kukongviriyapan V, Pakdeechote P, Prachaney P: Ellagic Acid Prevents L-NAME-Induced Hypertension via Restoration of eNOS and p47phox Expression in Rats. Nutrients 2015;7:5265-5280.

29 Han DH, Lee MJ, Kim JH: Antioxidant and apoptosis-inducing activities of ellagic acid. Anticancer Res 2006;26:3601-3606.

-30 Chang WC, Yu YM, Chiang SY, Tseng CY: Ellagic acid suppresses oxidised low-density lipoprotein-induced aortic smooth muscle cell proliferation: studies on the activation of extracellular signal-regulated kinase 1/2 and proliferating cell nuclear antigen expression. Br J Nutr 2008;99:709-714.

-31 Wang N, Wang ZY, Mo SL, Loo TY, Wang DM, Luo HB, Yang DP, Chen YL, Shen JG, Chen JP: Ellagic acid, a phenolic compound, exerts anti-angiogenesis effects via VEGFR-2 signaling pathway in breast cancer. Breast Cancer Res Treat 2012;134:943-955.

-32 Chang Y, Chen WF, Lin KH, Hsieh CY, Chou DS, Lin LJ, Sheu JR, Chang CC: Novel bioactivity of ellagic Acid in inhibiting human platelet activation. Evid Based Complement Alternat Med 2013;2013:595128.

-33 Yilmaz B, Usta C: Ellagic acid-induced endothelium-dependent and endothelium-independent vasorelaxation in rat thoracic aortic rings and the underlying mechanism. Phytother Res 2013;27:285-289.

-34 Carrillo-Sepulveda MA, Ceravolo GS, Furstenau CR, Monteiro Pde S, Bruno-Fortes Z, Carvalho MH, Laurindo FR, Tostes RC, Webb RC, Barreto-Chaves ML: Emerging role of angiotensin type 2 receptor (AT2R)/Akt/NO pathway in vascular smooth muscle cell in the hyperthyroidism. PLoS One 2013;8:e61982.

-35 Zhou J, Ni X, Huang X, Yao J, He Q Wang K, Duan T: Potential Role of Hyperglycemia in Fetoplacental Endothelial Dysfunction in Gestational Diabetes Mellitus. Cell Physiol Biochem 2016;39:1317-1328.

-36 Wu Y, Xue L, Du W, Huang B, Tang C, Liu C, Qiu H, Jiang Q: Polydatin Restores Endothelium-Dependent Relaxation in Rat Aorta Rings Impaired by High Glucose: A Novel Insight into the PPARbeta-NO Signaling Pathway. PLoS One 2015;10:e0126249. 


\section{Cellular Physiology Cell Physiol Biochem 2017;44:1174-1187 \begin{tabular}{l|l|l} 
DOI: 10.1159/000485448 & $\begin{array}{l}\text { (c) } 2017 \text { The Author(s). Published by S. Karger AG, Basel } \\
\text { www.karger.com/cpb }\end{array}$
\end{tabular}

Rozentsvit et al.: High Glucose-Induced Vascular Dysfunction and Vascular Protective Effect of Ellagic Acid

-37 Rani UP, Kesavan R, Ganugula R, Avaneesh T, Kumar UP, Reddy GB, Dixit M: Ellagic acid inhibits PDGF-BBinduced vascular smooth muscle cell proliferation and prevents atheroma formation in streptozotocininduced diabetic rats. J Nutr Biochem 2013;24:1830-1839.

38 Sahoo S, Meijles DN, Pagano PJ: NADPH oxidases: key modulators in aging and age-related cardiovascular diseases? Clin Sci (Lond) 2016;130:317-335.

-39 Quintela AM, Jimenez R, Piqueras L, Gomez-Guzman M, Haro J, Zarzuelo MJ, Cogolludo A, Sanz MJ, Toral M, Romero M, Perez-Vizcaino F, Duarte J: PPARbeta activation restores the high glucose-induced impairment of insulin signalling in endothelial cells. Br J Pharmacol 2014;171:3089-3102.

40 Taniguchi K, Xia L, Goldberg HJ, Lee KW, Shah A, Stavar L, Masson EA, Momen A, Shikatani EA, John R, Husain M, Fantus IG: Inhibition of Src kinase blocks high glucose-induced EGFR transactivation and collagen synthesis in mesangial cells and prevents diabetic nephropathy in mice. Diabetes 2013;62:38743886.

41 Hardigan T, Spitler K, Matsumoto T, Carrillo-Sepulveda MA: Activation of Toll-like receptor 3 increases mouse aortic vascular smooth muscle cell contractility through ERK1/2 pathway. Pflugers Arch 2015;467:2375-2385.

42 Matsumoto T, Ishida K, Nakayama N, Kobayashi T, Kamata K: Involvement of NO and MEK/ERK pathway in enhancement of endothelin-1-induced mesenteric artery contraction in later-stage type 2 diabetic GotoKakizaki rat. Am J Physiol Heart Circ Physiol 2009;296:H1388-1397.

43 Orasanu G, Plutzky J: The pathologic continuum of diabetic vascular disease. J Am Coll Cardiol 2009;53:S35-42.

44 Zurova-Nedelcevova J, Navarova J, Drabikova K, Jancinova V, Petrikova M, Bernatova I, Kristova V, Snirc V, Nosal'ova V, Sotnikova R: Participation of reactive oxygen species in diabetes-induced endothelial dysfunction. Neuro Endocrinol Lett 2006;27:168-171.

-45 Guzik TJ, Mussa S, Gastaldi D, Sadowski J, Ratnatunga C, Pillai R, Channon KM: Mechanisms of increased vascular superoxide production in human diabetes mellitus: role of NAD(P)H oxidase and endothelial nitric oxide synthase. Circulation 2002;105:1656-1662.

46 Evans JL, Goldfine ID, Maddux BA, Grodsky GM: Oxidative stress and stress-activated signaling pathways: a unifying hypothesis of type 2 diabetes. Endocr Rev 2002;23:599-622.

47 Carrillo-Sepulveda MA, Matsumoto T: Phenotypic modulation of mesenteric vascular smooth muscle cells from type 2 diabetic rats is associated with decreased caveolin-1 expression. Cell Physiol Biochem 2014;34:1497-1506.

48 Brunetti L, Kalabalik J: Management of type-2 diabetes mellitus in adults: focus on individualizing noninsulin therapies. P T 2012;37:687-696.

-49 Yusuf S, Dagenais G, Pogue J, Bosch J, Sleight P: Vitamin E supplementation and cardiovascular events in high-risk patients. The Heart Outcomes Prevention Evaluation Study Investigators. N Engl J Med 2000;342:154-160.

50 Lonn E, Yusuf S, Hoogwerf B, Pogue J, Yi Q Zinman B, Bosch J, Dagenais G, Mann JF, Gerstein HC, Study H, Study M-H: Effects of vitamin E on cardiovascular and microvascular outcomes in high-risk patients with diabetes: results of the HOPE study and MICRO-HOPE substudy. Diabetes Care 2002;25:1919-1927.

51 Pereira TM, Pimenta FS, Porto ML, Baldo MP, Campagnaro BP, Gava AL, Meyrelles SS, Vasquez EC: Coadjuvants in the Diabetic Complications: Nutraceuticals and Drugs with Pleiotropic Effects. Int J Mol Sci 2016;17.

52 Gorin Y, Wauquier F: Upstream regulators and downstream effectors of NADPH oxidases as novel therapeutic targets for diabetic kidney disease. Mol Cells 2015;38:285-296.

53 Xi G, Shen X, Maile LA, Wai C, Gollahon K, Clemmons DR: Hyperglycemia enhances IGF-I-stimulated Src activation via increasing Nox4-derived reactive oxygen species in a PKCzeta-dependent manner in vascular smooth muscle cells. Diabetes 2012;61:104-113.

54 Tyagi N, Moshal KS, Sen U, Vacek TP, Kumar M, Hughes WM, Jr., Kundu S, Tyagi SC: H2S protects against methionine-induced oxidative stress in brain endothelial cells. Antioxid Redox Signal 2009;11:25-33.

55 Takayama T, Wada A, Tsutamoto T, Ohnishi M, Fujii M, Isono T, Horie M: Contribution of vascular NAD(P) $\mathrm{H}$ oxidase to endothelial dysfunction in heart failure and the therapeutic effects of HMG-CoA reductase inhibitor. Circ J 2004;68:1067-1075. 


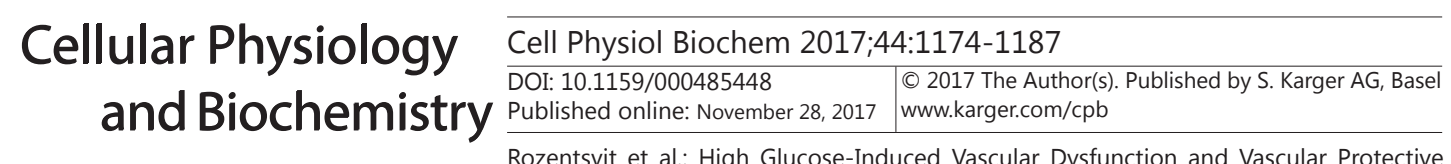
Effect of Ellagic Acid

56 Elsherbiny NM, El-Sherbiny M: Thymoquinone attenuates Doxorubicin-induced nephrotoxicity in rats: Role of Nrf2 and NOX4 Chem Biol Interact 2014;223:102-108.

-57 Vendrov AE, Vendrov KC, Smith A, Yuan J, Sumida A, Robidoux J, Runge MS, Madamanchi NR: NOX4 NADPH Oxidase-Dependent Mitochondrial Oxidative Stress in Aging-Associated Cardiovascular Disease. Antioxid Redox Signal 2015;23:1389-1409.

-58 Sedeek M, Callera G, Montezano A, Gutsol A, Heitz F, Szyndralewiez C, Page P, Kennedy CR, Burns KD, Touyz RM, Hebert RL: Critical role of Nox4-based NADPH oxidase in glucose-induced oxidative stress in the kidney: implications in type 2 diabetic nephropathy. Am J Physiol Renal Physiol 2010;299:F1348-1358.

59 Schramm A, Matusik P, Osmenda G, Guzik TJ: Targeting NADPH oxidases in vascular pharmacology. Vascul Pharmacol 2012;56:216-231.

60 Kim J, Seo M, Kim SK, Bae YS: Flagellin-induced NADPH oxidase 4 activation is involved in atherosclerosis. Sci Rep 2016;6:25437.

61 Lee CF, Qiao M, Schroder K, Zhao Q Asmis R: Nox4 is a novel inducible source of reactive oxygen species in monocytes and macrophages and mediates oxidized low density lipoprotein-induced macrophage death. Circ Res 2010;106:1489-1497.

62 Liu JC, Chen JJ, Chan P, Cheng CF, Cheng TH: Inhibition of cyclic strain-induced endothelin-1 gene expression by resveratrol. Hypertension 2003;42:1198-1205.

63 van Diepen JA, Thiem K, Stienstra R, Riksen NP, Tack CJ, Netea MG: Diabetes propels the risk for cardiovascular disease: sweet monocytes becoming aggressive? Cell Mol Life Sci 2016;73:4675-4684.

64 Aravindhan V, Madhumitha H: Metainflammation in Diabetic Coronary Artery Disease: Emerging Role of Innate and Adaptive Immune Responses. J Diabetes Res 2016;2016:6264149.

65 Kothari V, Galdo JA, Mathews ST: Hypoglycemic agents and potential anti-inflammatory activity. J Inflamm Res 2016;9:27-38.

-66 BenSaad LA, Kim KH, Quah CC, Kim WR, Shahimi M: Anti-inflammatory potential of ellagic acid, gallic acid and punicalagin A\&B isolated from Punica granatum. BMC Complement Altern Med 2017;17:47.

67 Seo CS, Jeong SJ, Yoo SR, Lee NR, Shin HK: Quantitative Analysis and In vitro Anti-inflammatory Effects of Gallic Acid, Ellagic Acid, and Quercetin from Radix Sanguisorbae. Pharmacogn Mag 2016;12:104-108.

68 Guo R, Li W, Liu B, Li S, Zhang B, Xu Y: Resveratrol protects vascular smooth muscle cells against high glucose-induced oxidative stress and cell proliferation in vitro. Med Sci Monit Basic Res 2014;20:82-92.

69 Yang J, Chen L, Ding J, Fan Z, Li S, Wu H, Zhang J, Yang C, Wang H, Zeng P, Yang J: MicroRNA-24 inhibits high glucose-induced vascular smooth muscle cell proliferation and migration by targeting HMGB1 Gene 2016;586:268-273.

70 Sun A, Wang Y, Liu J, Yu X, Sun Y, Yang F, Dong S, Wu J, Zhao Y, Xu C, Lu F, Zhang W: Exogenous H2S modulates mitochondrial fusion-fission to inhibit vascular smooth muscle cell proliferation in a hyperglycemic state. Cell Biosci 2016;6:36.

71 Ganguly R, Sahu S, Chavez RJ, Raman P: Trivalent chromium inhibits TSP-1 expression, proliferation, and O-GlcNAc signaling in vascular smooth muscle cells in response to high glucose in vitro. Am J Physiol Cell Physiol 2015;308:C111-122.

-72 Shimabukuro M, Higa N, Chinen I, Yamakawa K, Takasu N: Effects of a single administration of acarbose on postprandial glucose excursion and endothelial dysfunction in type 2 diabetic patients: a randomized crossover study. J Clin Endocrinol Metab 2006;91:837-842. 